\title{
Loss Differentiated Rate Adaptation in Wireless Networks
}

\author{
Shaoen Wu, Saâd Biaz \\ \{wushaoe,sbiaz\}@eng.auburn.edu \\ Auburn University \\ Dept. of Computer Science and Software Engineering \\ 107 Dunstan Hall, Auburn University, AL, 36849-5347, USA \\ Technical Report CSSE07-02 (January 15, 2007)
}

\begin{abstract}
Data rate adaptation is the process to select the optimal data rates for instantaneous channel conditions. This paper proposes a data rate adaptation technique that poses following traits: (1) it uses the periodical IEEE 802.11 beacons; (2) moreover, it distinguishes packet losses due to channel fading from those due to collisions and take actions appropriate for each type of loss; (3) in addition, it introduces the lowest data rate retransmission for the very first retransmission after packet loss. In traditional strategies, after a packet loss, retransmissions are often attempted with the same data rate. This work analytically shows that retransmitting at the lowest data rate is more efficient, especially in poor SNR environment or when there is no knowledge of the loss cause (channel degradation or transmission collision). This scheme, dubbed Loss Differentiated Rate Adaptation (LDRA), is extensively evaluated through simulations and shown to perform better especially when network collision is heavy.
\end{abstract}

Keyword: Rate Adaptation; Loss Differentiation; Beacon.

\section{INTRODUCTION}

The wireless medium is exposure and highly sensitive to environment disturbances (e.g., weather conditions, interference from other sources...). Such a sensitivity renders the wireless medium highly instable and results in fluctuations of wireless channel quality. This instability that wireless channel experiences calls for data adaptation algorithms to dynamically select appropriate data rate (modulation schema) upon different levels of channel quality.

Traditional data adaptation strategies, like [1]-[7], have been proposed to successfully estimate instantaneous data rate for wireless channel by analyzing either SNR or packet loss rate. But, the rate adapted is not completely accurate all the time due to the fluctuation of the channel and/or the mobility of network. Rate mismatch might lead to packet loss. Summarily, wireless packet transmission with contention access protocols may be impacted by two general influences: (a) wireless signal degradation due to further distance; (b) or the packet collision from access contention. Differentiation of these two categories of causes makes data adaptation perform differently. As for channel degradation, more robust modulation (with low data rate) can increase SNR and counter degradation. But, in case of collision, the sacrifice of data rate can not alleviate the problem that wireless channel suffers. With our best knowledge, this is little rate adaption work concerning loss differentiation ${ }^{1}$. In this work, we propose a passive sender-based data rate adaptation strategy with loss differentiation without introducing extra cost.

Our work has following three key contributions. First, before transmitting, a station estimate the channel quality by monitoring periodic mandatory beacon frame. Second, when the station experiences packet loss, our schema (LDRA) differentiates packet loss causes with proposed lowest rate retransmission. Data rate is not decreased for collision loss without unnecessary sacrifice of performance. Although rate is decreased for channel degradation loss, the binary exponential backoff is turned off in such case because collision does not happen. It should be pointed out that this differentiation algorithm does not depend on the beacon monitoring and it can apply to both sender-based and receiver-based data adaptations. Finally, with 802.11 environment, we analytically demonstrate that the lowest rate retransmission does not certainly do harm to network performance, especially in poor SNR environment.

The remaining of this paper is organized as follows: Section II describes related work in data rate adaptation. Afterward, Section III describes four motivations behind our work. Section IV details the proposed scheme. We justify the lowest rate retransmission in Section V. The simulation results are presented in section VI. Section VII concludes this paper.

\section{RELATED WORK}

Rate adaptation schemas can be either sender based or receiver based; packet loss rate driven or signal strength (RSSI) driven; loss differentiated or non loss differentiated. Different rate adaptation strategy can be categorized into different class.

\footnotetext{
${ }^{1}$ When this work was in draft, there were other two papers considering collision published: Kim, et al, INFOCOM06 and Wong, et al, MOBICOM06.
} 


\begin{tabular}{|c|c|c|c|c|}
\hline Schema & Based & Driven & RTS/CTS & $\begin{array}{l}\text { Loss Differentia- } \\
\text { tion }\end{array}$ \\
\hline ARF & Sender & Loss rate & & \\
\hline Pang & Sender & Loss rate & & $\checkmark$ \\
\hline RBRA & Receiver & RSSI & $\checkmark$ & \\
\hline RRAA & Sender & Loss rate & $\checkmark$ & $\checkmark$ \\
\hline CARA & Sender & Loss rate & $\checkmark$ & $\checkmark$ \\
\hline LDRA & Sender & RSSI & & $\checkmark$ \\
\hline
\end{tabular}

TABLE I

RAte AdAPTION Schemas

Auto Rate Fallback (ARF) [3] implemented in Lucent WaveLan-II product is almost the earliest proposed sender based scheme for data rate adaptation in WLAN. ARF is a loss rate driven schema. In ARF, a sender starts transmitting at the lowest data rate. If the transmission succeeds for ten consecutive packets, the sender increases its transmission data rate. Otherwise, it decreases the data rate. However, with ARF the data rate oscillates, and never converges to a stable data rate even under fairly steady channel conditions. This problem is straightforward from figure 3: although wireless channel can support 5.5 Mbps steadily, it fluctuates between 5.5 Mbps and $11 \mathrm{Mbps}$. Under such condition, the resulting packet loss rate would be not less than $16 \%$.

Pang and Liew [8] proposed an extension to Auto Rate Fallback (ARF) with integration of loss differentiation. This work exploits a loss-differentiating MAC layer technique [9] by the same authors. This differentiation technique assumes that the packet header is short and thus resistant to wireless channel fading. Therefore, if the packet header can be decoded while the payload is corrupted, this loss-differentiating technique infers that the packet loss is due to wireless channel degradation. Otherwise, if all the frame is lost, it infers that the packet loss results from a collision. In this work [8], "the data rate is reduced only when a loss of data frame is caused by link errors". However, the authors do not suggest how to react to packet loss from collision and how to deal with retransmissions after a packet loss.

Holland, Vaidya, and Bahl proposed the Receiver Based Rate Adaptation scheme (dubbed RBRA) [10]. Based on channel conditions sensed through the reception of RTS, the receiver determines the most appropriate data rate that the channel can support and feeds back the estimated rate in the CTS message. The sender uses this estimated rate for data exchange. This proposal works for both WLAN and multi-hop ad hoc networks only if control (probe) frames such as RTS/CTS are used prior to data exchange. RBRA assumes that channel conditions do not vary much between the time the RTS is received and the data packet is sent.

At the moment our this work was in progress, two proposals considering loss differentiation in IEEE 802.11 networks were published. The first is called Robust Rate Adaptation Algorithm (dubbed RRAA) by Wong, et al. RRAA is also a loss rate driven schema. It keeps short term frame loss rate, and adapts the transmission rate accordingly. Beyond that, the authors also proposed "Adaptive RTS Filter" to assess new channel condition. When a certain number of frames are lost without RTS/CTS probing frame, the RTS frame is triggered to estimate channel quality. The authors argue that "Adaptive RTS Filter" is helpful to "prevent collision losses from triggering rate decrease". The other strategy is proposed by Kim, et al, named CARA. CARA requires RTS/CTS probing. If data frame fails without RTS, RTS probing is turned on. This algorithm assumes all RTS transmission failure is caused only by collision. Therefore, if RTS is transmitted successfully, but data packet transmission fails, the failure is inferred from channel degradation. Only in such channel fading is the data rate decreased as in ARF. Otherwise, data rate is not changed.

The table I characterizes our proposed LDRA and those above rate adaptation algorithms.

\section{Motivation}

\section{A. Motivation $\sharp 1$ : Initial Rate Estimation}

In IEEE 802.11, mobile stations are grouped as Basic Service Set (BSS) in infrastructure network or Independent Basic Service Set (IBSS) in infrastructureless network. The periodic Beacon frame in IEEE 802.11 is used for the following purposes [11]:

- Synchronization between wireless stations or between wireless station and access point.

- Power management to save power in case there is no traffic.

- Indication of multiple rates supported for in wireless channel with different modulations

From above, the beacon is an essential component in either infrastructure (WLAN) or infrastructureless networks (Ad-Hoc). This frame is periodically broadcast.

Sender based schemes might suffer from their inability to appropriately select a data rate for the very first frame(e.g., just after some long inactivity on the channel). After inactivity, channel conditions may have degraded and a sender may inefficiently send the first frame multiple times at an inappropriate data rate before adjusting the data rate. The mandatory periodic beacon frame provides opportunity for the initial rate estimation without any extra effort. 


\section{B. Motivation $\sharp 2$ : Request of Loss Differentiation}

In traditional data rate adaptation schemas, a packet loss is generally and unduly considered to indicate that wireless channel conditions degraded. In fact, a packet loss may also be due to transmission collisions: data rate should be decreased in response to channel conditions degradation, but not for collisions. Decreasing data rate for all losses without differentiation incurs underutilization of network resources. Also, a station suffering packet loss from channel degradation is not necessary to back off binary exponentially, because it does not collide with other stations. Different behavior upon different frame loss calls for loss differentiation.

\section{Motivation $\sharp 3$ : Necessity of Sender-based Schema}

The RTS/CTS control frames are optional in standard IEEE 802.11. If the size of data frames is small, as is the case for real time applications like VoIP, the use of RTS/CTS dramatically increases the relative overhead. Based on an analysis by Garg and Kappes [12], the use of RTS/CTS control frames yields an efficiency as low as 12\% if a unique station is transmitting VoIP traffic with 160 byte payload. So, RTS/CTS control frames should be used only when data frame size is over some threshold. In case RTS/CTS control frames are not used, all receiver based data rate adaptation schemes cannot be used.

In IEEE 802.11, optional RTS/CTS control frames ought to be transmitted at the lowest data rate so that they can reliably be received by each station in transmission range. Li et al. (Full Auto Rate) [6] showed that higher throughput can be achieved if the RTS/CTS frames are also transmitted at a higher estimated data rate based on channel conditions. Thus, an accurate estimated data rate at the sender can improve the transmission of RTS/CTS frames and thereof the entire network performance.

\section{Motivation $\sharp 4$ : Retransmission Cost Reduction}

In state-of-the-art rate adaptation algorithms, the retransmission after a packet loss is normally performed at the same data rate. Such a strategy is not adequate and essentially inefficient, if the packet loss resulted from channel degradation. In such cases, transmissions at the same rate only results in repeated packet loss and wastes precious network bandwidth. If the retransmission is performed at the lowest data rate directly, it succeeds if the receiver station is still in radio range, although the channel condition faded. By this way, network cost from retransmission can be substantially reduced.

\section{Loss DifFERENTIATION RATE AdAPTATION}

The proposed scheme, dubbed LDRA in this work, mainly consists of three components: (1) data rate estimation for initial and ongoing transmissions using received signal strength (RSS), (2) data rate selection for retransmissions after packet loss, and (3) packet loss differentiation with corresponding strategies. Note that LDRA works for IEEE 802.11 in infrastructure mode as well as for infrastructureless (ad hoc) mode.

Rate Estimation: In IEEE 802.11 infrastructure network, a station that is associated and synchronized with its access point knows the beacon period. Each station periodically listens for a beacon frame that can be used to measure the channel conditions through the signal-to-noise ratio (SNR), the received signal strength (RSS), the packet loss rate, or the error bit rate. Based on such collected information, the station can estimate the most appropriate data rate to send a frame to the source of the beacon. The accuracy of such estimation depends on the beacon interval. It should be quite accurate because the beacon period is small and channel conditions would not drastically change within such a short interval. This should be especially true for low speed network or stationary scenarios like mesh networks. Even if the beacon interval is comparably large, this estimated rate is still more appropriate than a randomly selected or "guessed" the initial data rate at the beginning of the transmission.

An supplement to the beacon estimation is to take all communication packets into consideration for adaptive rate adaption after two stations starts their communication. Such a strategy can provide more accurate estimation if there are multiple transmissions between two consecutive beacons.

When RSS is collected from physical layer, LDRA adopts the data rate adaptively from following formula: $R_{n}=\alpha *$ $R_{n-1}+(1-\alpha) * r$ where $R_{n}$ is the $n^{\text {th }}$ adapted rate, and $r$ is the instantaneous rate estimated from the RSS of received packet. When $\alpha$ is a constant, this equation is similar to the one in Pavon and Choi [5]. We also simulate the adaptive $\alpha$ as in: $\alpha=\beta * \frac{R_{n}-R_{\text {low }}}{R_{\text {high }}-R_{\text {low }}} ; \beta$ is a constant valuated from 0 to $1 ; R_{\text {low }}$ is the RSS low threshold for some specific data rate (e.g. $5.5 \mathrm{Mbps}) ;$ and $R_{\text {high }}$ is the RSS high threshold for the same data rate.

Packet Loss Differentiation: Since no data rate adaptation scheme can completely accurately select the most appropriate data rate for all transmissions all the time, the sender may transmit at a data rate that is too high, leading to frame losses. When a frame is lost, no acknowledgement is fed back to sender. The absence of an acknowledgement can hint to decrease the data rate. In ARF [3] and other packet loss based rate adaptation schemes, the number of lost packets or the packet loss rate determine the data rate. In ARF, if there are two consecutive packet losses, the data rate is decreased, no matter what caused the packet loss. However, a lower rate would unduly impact the performance if the packet loss was due to a collision. Thus, it is critical to determine the cause of a packet loss and take appropriate actions for data rate adaptation. 


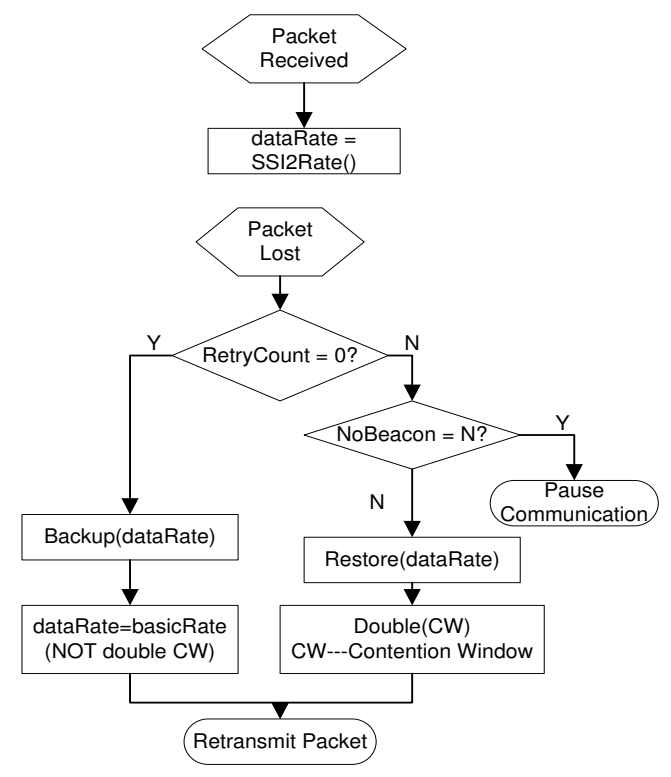

Fig. 1. LDRA Algorithm Flow Chart

LDRA introduces the lowest rate retransmission to differentiate packet loss. When a packet loss occurs (no acknowledgement received at the sender) for its very first time, the sender retransmits the frame directly at the lowest data rate, instead of the same rate. Such retransmission is beneficial for loss differentiation as illustrated in the following scenarios:

- If the wireless channel did not further degrade, then the receiver should be able to receive this retransmitted data packet (due to lowest data rate). Thus the sender can receive the acknowledgement and accordingly adapt the data rate to a higher level.

- If the retransmitted packet is still lost (no acknowledgement), and no beacon is received during the latest $N$ beacon periods, the sender can infer that the receiver is out of range (for the current channel quality). The sender should pause its transmissions until it can again detect a beacon frame. This results in two benefits: one is to reduce network traffic and therefore reduce unnecessary collisions (or hidden/exposed terminal problems). The other is to save power as the power issue is a critical limitation for mobile stations.

- If the retransmission at the lowest data rate is also lost (no acknowledgement), but a beacon frame has been received in the latest $N$ beacon periods, the sender can infer that the loss is rather due to collisions. Therefore, the sender can invoke the binary exponential back off and maintain a higher data rate (since losses appear to be due to collisions).

Operations on Frame Loss: Three operations are proposed to improve network performance. The first recommended is that the sender should not double its contention window as in IEEE 802.11 [11] immediately in its first retransmission at the lowest data rate. The rationale behind such a change is that the binary exponential backoff of the contention window in CSMA/CA [13] is meant to react to collisions, not to channel degradation. When frame is lost for the first time, there is no knowledge of the frame loss root cause. Frame losses in multi-rates wireless networks are not always related to collisions.

The second operation is for frame loss from channel degradation. In such case, the receiver station should be able to decode the lowest rate retransmission frame. Then, it estimates the new rate and feeds back the new rate selected in the CTS or ACK frame as in RBRA. Also, the sender can assess channel quality from the CTS or ACK from receiver. By such way, the sender can adapt its new rate immediately after its very first rate retransmission at lowest rate, and thus avoids the performance loss from the lowest data rate for further transmissions.

The third one is suggested in case frame loss is identified from collision. If frame fails due to collision, lower data rate can not alleviate the problem. Instead, it increases radio coverage and worsens the collision. Therefore, the data rate should keep unchanged for successive transmissions. This is the critical difference from traditional rate adaptation algorithms without loss differentiation.

The LDRA core algorithm is illustrated by the flow chart Figure 1.

\section{JUSTIFICATION OF THE LOWEST RATE RETRANSMISSION}

In IEEE 802.11b [14], there are four modulation schemes: BPSK (1Mbps), QPSK (2Mbps), CCK5.5 (5.5Mbps), CCK11 (11Mbps). We analyze the expected normalized transmissions for a packet to be transmitted successfully with each modulation in different SNR environment. 


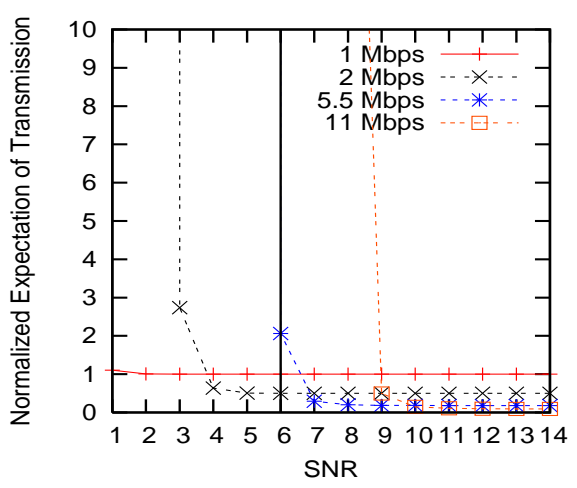

Fig. 2. Normalized Expected Transmission

In IEEE 802.11, the FER (Frame Error Rate) is associated with packet length of 1024 bytes. If BER (Bit Error Rate) $p$ is very small and losses are independents, then the corresponding FER should be about $p * 1024 * 8$. The expected transmissions for a packet to be transmitted successfully should be $\frac{1}{1-F E R}$. Considering the same transmission at different rate takes different time, the expected transmission normalized by rate for a successful packet should be $\frac{1}{(1-F E R) * r a t e}$. Based on BER data in the work by $\mathrm{Wu}$ [15], Figure 2 illustrates the relationship between normalized expected transmission and SNR for different modulations. The $x$-axis represents SNR, and the $y$-axis is the normalized expected transmission. Bianchi, et al. [16] investigated that most of time the SNR in outdoor environment is not large than $6 \mathrm{db}$ for $802.11 \mathrm{~b} / \mathrm{g}$. From this figure, it can be observed that, if a packet is transmitted at $5.5 \mathrm{Mbps}$ or $11 \mathrm{Mbps}$ in an environment with SNR less than $6 \mathrm{db}$, it has to be retransmitted so many times. In other words, transmissions are unlikely to succeed. But for the lowest rate of 1 Mpbs or 2 Mbps, a packet can successfully be transmitted almost every time in very low SNR. Thus, in traditional retransmission scheme, even if a packet is lost from channel fading with a very low SNR, CSMA/CA always assumes the loss to be due to collision. It backs off and retransmits the packet at the same high data rate again for several times. As we can see from the above figure, this retransmission process is not promising at all for a packet loss resulting from channel fading. Thus, it is important to retransmit at the lowest data rate and differentiate the reason of a packet loss.

\section{EXPERIMENTS AND SimULATIONS}

Simulation configuration: LDRA is simulated in WLAN mode with 802.11b on ns-2 [17] (version 2.29). The proposed scheme is compared with ARF [3] and adaptive auto rate [5] through simulations of a single flow and then multiple competing flows. Only three levels of data rates are used: $11 \mathrm{Mbps}, 5.5 \mathrm{Mbps}$ and $2 \mathrm{Mbps}$. Wireless channel fading is modeled as twoway-ground model.

Data Rate Adaptation: First, LDRA is compared with ARF. The first experiment consists of one mobile node with one access point. The node moves around such that $5.5 \mathrm{Mbps}$ data rate is the most appropriate. Figure 3 plots the results: the $x$-axis represents the time and the $y$-axis is the data rate. Figure 3 shows, as expected, that ARF frequently changes the data rate because it blindly makes adjustments regardless of wireless channel conditions. Under the same conditions, the proposed scheme, LDRA, remains steady at the appropriate data rate of 5.5 Mbps.

LDRA is also evaluated on a network with multiple nodes. The purpose of this experiment is to evaluate the retransmission at the lowest rate after a frame loss due to a collision (rather than wireless channel fading). Figure 4 , shows the case where the retransmission is performed at the lowest data rate $(2 \mathrm{Mbps})$ after a packet loss occurring at a high data rate. Since the packet loss is due to a collision, the data rate is maintained at the same level, unlike in ARF where multiple retransmissions occur at the next lower data rate. The retransmissions in LDRA at the lowest data limits the number of retransmissions to one.

Throughput Improvement: We define throughput improvement of LDRA over some scheme $X$ as $\frac{\text { Throughput } L D R A-\text { Throughput }_{X}}{\text { Throughput }_{X}}$.

Figure 5 plots the throughput improvement for an experiment with varying the density of moving nodes at different velocities in WLAN network. The $x$-axis is for velocity and the $y$-axis represents the throughput improvement for the entire network. As shown on the figure, the velocity does not significantly impact the improvement. But the improvement sharply increases with nodes density. This can be explained by the ability of LDRA to correctly distinguish the real cause of a packet loss between a collision and a wireless channel fading. As collisions increase with nodes density, mobile nodes with LDRA correctly diagnose collision losses for which they maintain the original data rate. With ARF, the node unduly decreases the data rate. As collisions increase, LDRA performs better than ARF, leading to a better throughput improvement. The relationship between throughput improvement and network density is stronger from the simulation results reported in Figure 6 . In this scenario, the mobile nodes move at random velocity in Ad-Hoc network. The $x$-axis and $y$-axis respectively represent the number of mobile nodes in network and the throughput improvement. 


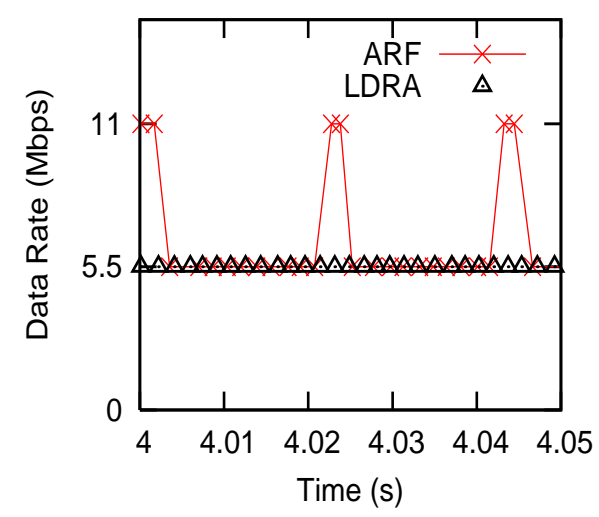

Fig. 3. Rate Adaptation Comparison

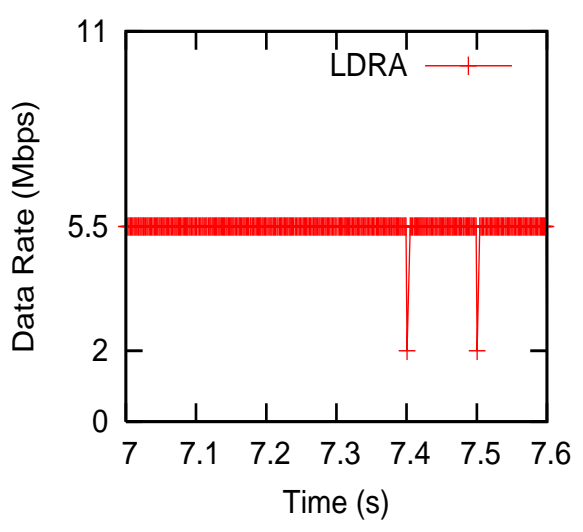

Fig. 4. LDRA Rate Adaptation with Collision

We also compare the throughput from LDRA with that from work by Pavon and Choi [5] in Ad-Hoc network. Figure 7 present the results. Note that the benefits from LDRA continuously increase as the network density increases. In low network density, the adaptive scheme [5] performs better than LDRA, because it adapts rate more frequently than the beacon frequency imposed on LDRA. But in a high node density environment, LDRA overperforms it, due to the ability to differentiate loss.

Another set of experiments were carried with TCP flows. Different numbers of TCP flows are tested in different scenarios. The total network throughput for all TCP flows is used to measure the improvement. In simulation result Figure 8 , the $x$-axis represents the number of TCP flows. The $y$-axis represents the entire network throughput improvement of LDRA over ARF. This figure shows a dramatic improvement up to almost $100 \%$ for different scenarios.

Delay Jitter Improvement: Figure 9 shows a significant improvement of delay jitter in WLAN by LDRA over ARF. Delay jitter here is defined as the time difference between two successive packets delays. We collect the maximum delay jitter

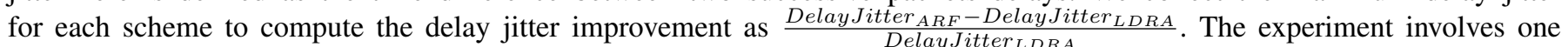
mobile node and its access point with UDP flow. The $x$-axis and $y$-axis respectively represent the velocity and the delay

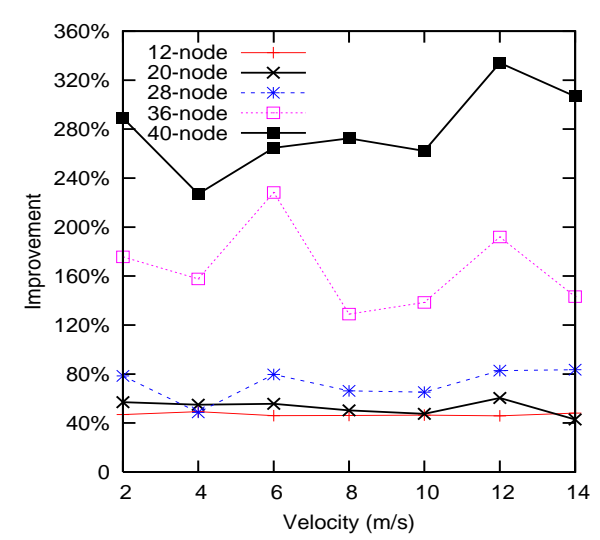

Fig. 5. Throughput Improvement at Different Velocity 


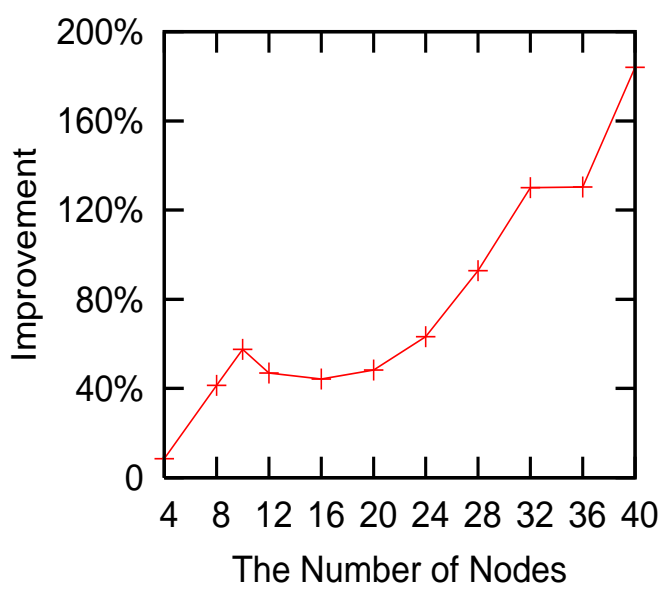

Fig. 6. Improvement with Network Density

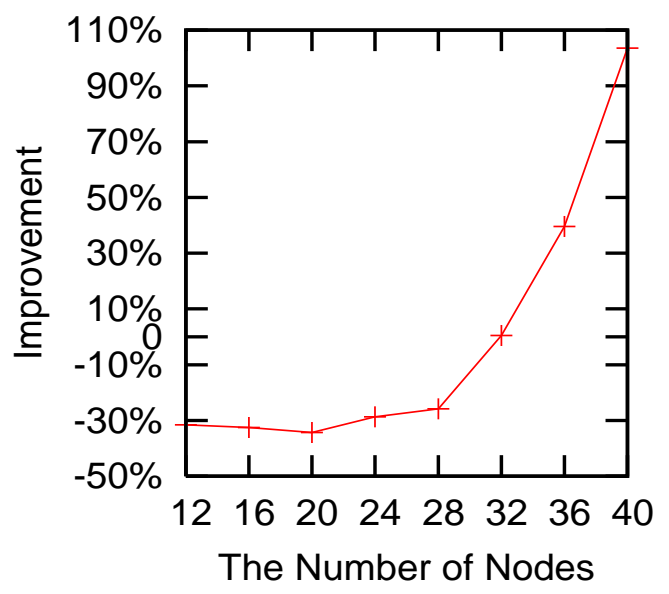

Fig. 7. Improvement with LDRA vs Adaptive

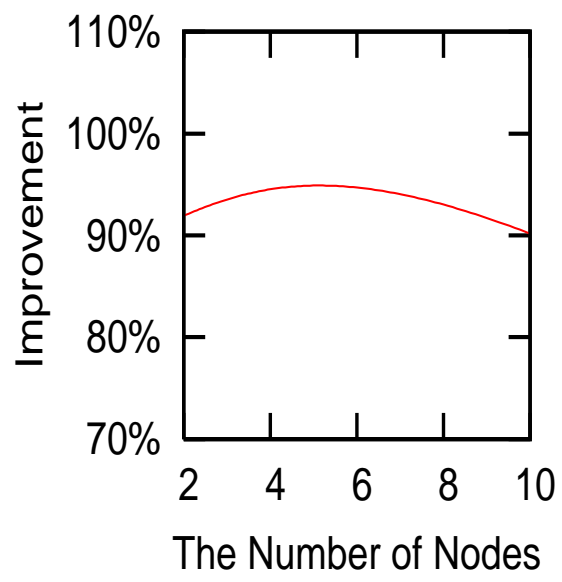

Fig. 8. Throughput Improvement in TCP 


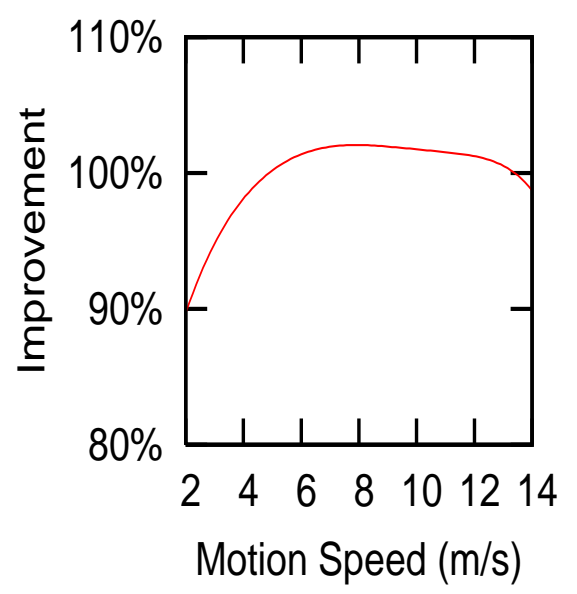

Fig. 9. Delay Jitter Improvement

jitter improvement. Retransmissions at the lowest data rate greatly contribute to this improvement because the number of retransmissions is minimized by LDRA. LDRA, with such a strategy, introduces less variability of delay after a packet loss and therefore yields a lower delay jitter than ARF.

\section{CONCLUSION}

This work presented a Loss Differentiated Rate Adaptation (LDRA) scheme to dynamically adjust data rate in a wireless network. LDRA measures channel conditions through the IEEE 802.11 periodic beacons and determines the most appropriate data rate. Channel conditions allow LDRA to correctly discriminate packet losses due to corruption from those due to collisions. For losses not related to collisions, the mobile node does not double its contention window. This ability to discriminate significantly improves throughput. Additionally, retransmissions at the lowest data rate minimizes the expected number of retransmissions after a loss and reduce both the delay and the delay jitter.

\section{REFERENCES}

[1] M. M. M. Lacage and T. Turletti, "IEEE 802.11 Rate Adaptation: A Practical Approach," in MSWiM04, 2004.

[2] A. S. B. Sadeghi, V. Kanodia and E. Knightly, "Opportunistic Media Access for Multirate Ad Hoc Networks," in MOBICOMO2, 2002.

[3] A. Kamerman and L. Monteban, "WaveLAN II: A high-performance wireless LAN for the unlicensed band," Bell Labs Technical Journal, pp. 118-133, 1997.

[4] D. Qiao, S. Choi, and K. Shin, "Goodput Analysis and Link Adaptation for IEEE 802.11a Wireless LANs," IEEE TRANSACTIONS ON MOBILE COMPUTING, vol. 1, pp. 278-292, 2002.

[5] J. Pavon and S. Choi, "Link Adaptation Strategy for IEEE 802.11 WLAN via Received Signal Strength Measurement," in ICC, 2003.

[6] Z. Li, A. Das, A. Gupta, and S. Nandi, "Full auto rate MAC protocol for wireless ad hoc networks," IEE Procedings on Communication, vol. 3, pp. 311-319, 2005.

[7] S. Wu and S. Biaz, "Exploring Beacon and Sender based Rate Adaptation in Wireless Networks," Auburn University, Tech. Rep., 2006.

[8] V. L. Qixiang Pang and S. C. Liew, "A Rate Adaptation Algorithm for IEEE 802.11 WLANs Based on MAC-Layer Loss Differentiation," in IEEE BROADNETS 2005 - Broadband Wireless Networking Symposium, Boston, USA, 2005, pp. 709-717.

[9] S. C. L. Q. Pang and V. Leung, "Design of an Effective Loss-Distinguishable MAC Protocol for 802.11 WLAN," IEEE Communications Letters, vol. 9, pp. 781-783, 2005 .

[10] G. Holland, N. Vaidya, and P. Bahl, “A rate-adaptive MAC protocol for multi-hop wireless networks," in In Proceedings of ACM MOBICOM'01, 2001.

[11] IEEE802.11, "http://standards.iee.org/getieee802/ $\quad$ download/802.11-1999.pdf," $1999 . \quad$ http://standards.ieee.org/getieee802/download/802.11-1999.pdf

[12] S. Garg and M. Kappes, "An Experimental Study of Throughput for UDP and VoIP Traffic in IEEE 802.11b Networks," in wcnc2003, 2003.

[13] S. S. Vaduvur Bharghavan, Alan Demers and L. Zhang, "MACAW: a media access protocol for wireless LAN's," in Proceedings of the conference on Communications architectures, protocols and applications, London, United Kingdom, 1994, pp. 212 - 225.

[14] IEEE802.11, "http://standards.ieee.org/getieee802/ $\quad$ download/802.11b-1999.pdf," $1999 . \quad$ Available: http://standards.ieee.org/getieee802/download/802.11b-1999.pdf

[15] X. Wu, "Simulate 802.11b Channel within NS2," National University of Singapore, Tech. Rep.

[16] F. F. Giuseppe Bianchi and D. Giustiniano, "802.11b/g Link Level Measurements for an Outdoor Wireless Campus Network," in Proceedings of the 2006 International Symposium on a World of Wireless, Mobile and Multimedia Networks (WoWMoM'06), 2006.

[17] NS2, "http://www.isi.edu/nsnam/ns/." [Online]. Available: http://www.isi.edu/nsnam/ns/ 